\title{
Trauma abdominal: experiencia de 4961 casos en el occidente de México
}

\author{
Abdominal trauma: experience of 4961 cases in Western Mexico \\ Gustavo Parra-Romero, Gabriel Contreras-Cantero, Diego Orozco-Guibaldo, Andrea Domínguez-Estrada, \\ José de Jesús Mercado-Martín del Campo y Luis Bravo-Cuéllar* \\ Servicio de Cirugía Medicina Legal, Hospital Civil de Guadalajara Fray Antonio Alcalde, Guadalajara, Jalisco, México
}

\begin{abstract}
Resumen
Introducción: El trauma es una de las principales causas de morbimortalidad en el mundo. El abdomen es, en frecuencia, la tercera región anatómica más afectada, y el compartimento intraabdominal es un sitio de hemorragia difícil de identificar, por lo que cobra importancia el conocimiento de las posibles lesiones tras un traumatismo. Objetivos: Describir y analizar el perfil sociodemográfico y las lesiones encontradas en pacientes con trauma abdominal en un hospital de referencia del occidente de México. Método: Se seleccionaron para su análisis los pacientes incluidos en el registro hospitalario local GDL-SHOT. Resultados: De 4961 pacientes, el 91.4\% fueron hombres, con un promedio de edad de 28.7 años. Respecto al mecanismo, el $39.7 \%$ correspondió a arma blanca, el 33\% a trauma cerrado y el $27.3 \%$ a arma de fuego. Los órganos más afectados fueron el intestino delgado (20.9\%), el hígado (18.2\%) y el colon (14.2\%). La estancia hospitalaria promedio fue de 6.95 días, con una mortalidad del 6.74\%. Conclusiones: En México, el trauma abdominal representa una causa importante de morbimortalidad, en especial en pacientes jóvenes, y predomina el mecanismo penetrante; el manejo más común es no conservador. La frecuencia de lesiones encontradas es discordante con la literatura de otros países y predominan las de vísceras huecas, probablemente por la diferencia en los mecanismos implicados.
\end{abstract}

PALABRAS CLAVE: Epidemiología. Laparotomía. Lesiones. México. Trauma abdominal.

\begin{abstract}
Introduction: Trauma is a leading cause of morbimortality in the world. Intraabdominal compartment is the third most affected anatomical region and bleeding from this origin is difficult to identify, therefore the importance to predict possible lesions to the abdominal cavity. Objective: To describe and analyze the sociodemographic profile and injuries found in patients with abdominal trauma in a western hospital in Mexico. Method: Consecutive patients included in the local registry GDL-SHOT were analyzed. Results: From 4961 patients, 91.4\% were men, with a mean age of 28.7 years. Regarding the mechanism of trauma, $39.7 \%$ were stab wounds, $33 \%$ blunt abdominal trauma and $27.3 \%$ gunshots. The most affected organs were: small bowel (20.9\%), liver (18.2\%), and colon (14.2\%). The mean hospital stay was 6.95 days with a mortality of $6.74 \%$. Conclusion: In Mexico, abdominal trauma represents an important cause of morbidity and mortality, especially in young patients. We found an important amount of penetrating trauma.
\end{abstract}

KEY WORDS: Abdominal injuries. Epidemiology. Laparotomy. Mexico. Wounds and injuries.

\author{
Correspondencia: \\ *Luis Bravo-Cuéllar \\ Hospital 278 \\ Coronel Calderón, 777 \\ Col. El Retiro \\ C.P. 44280 , Guadalajara, Jal., México \\ E-mail: Ibravoc9@outlook.com \\ Fecha de recepción: 27-06-2018 \\ Fecha de aceptación: 28-07-2018 \\ DOI: 10.24875/CIRU.18000509
}

Cir Cir. 2019;87:183-189

Contents available at PubMed www.cirugiaycirujanos.com 


\section{Introducción}

Las lesiones por trauma representan actualmente una de las principales causas de morbimortalidad en el mundo. Son un problema de salud pública de primera importancia, con altos costos por la pérdida de vidas humanas, la atención médica de estos pacientes $\mathrm{y}$, a largo plazo, la pérdida de productividad ${ }^{1,2}$. Además, dado el perfil demográfico de los pacientes afectados, el trauma es una de las principales causas de años de vida perdidos y de años vividos con discapacidad $^{2,3}$. Aunque se trata de un problema global, es bien conocido que, por diversos factores, las lesiones por trauma son más prevalentes en los países en desarrollo que carecen de un sistema de salud robusto para hacer frente a su tratamiento, por lo que el $90 \%$ de las muertes por trauma en el mundo ocurren en este contexto ${ }^{4}$.

En México, las lesiones por trauma han representado un monto importante de muertes en décadas previas, situación que se ha agudizado en los últimos años ${ }^{5,6}$. Según el Instituto Nacional de Estadística, Geografía e Informática, en 2015, los accidentes y agresiones representaron la cuarta y la octava causas de muerte, respectivamente, en la población general. Sin embargo, este dato es en especial relevante en los adultos jóvenes, pues representó el $50 \%$ de la mortalidad entre los 15 y los 34 años, es decir, la primera y la segunda causas de muerte en este grupo de edad. En 2016, se registraron 72,821 muertes violentas, de ellas el $51 \%$ accidentales ${ }^{7}$.

El abdomen es una de las regiones anatómicas más afectadas por las lesiones traumáticas y está involucrado en el $31 \%$ de los pacientes politraumatizados ${ }^{8}$, por lo que la identificación de las lesiones intraabdominales es crucial a fin de disminuir la morbimortalidad de este origen.

Dentro del abordaje del paciente con traumatismo abdominal deben considerarse factores que potencialmente pueden modificar el pronóstico, tales como el mecanismo del trauma, el agente de la lesión, las regiones anatómicas afectadas, el tiempo desde el suceso, el estado hemodinámico y neurológico del paciente, los hallazgos en la exploración física y los recursos disponibles en el lugar de la evaluación primaria ${ }^{6,9}$.

Respecto al mecanismo del trauma, se clasifica en cerrado (accidentes automovilísticos, accidentes peatonales, caídas y agresiones) o penetrante (agresiones con arma de fuego, arma blanca u objetos punzocortantes) ${ }^{10}$. La mayoría de los ingresos hospitalarios por trauma en los países desarrollados son secundarios a accidentes automovilísticos ${ }^{1,11,12}$; sin embargo, la incidencia de trauma penetrante ha aumentado en años recientes ${ }^{13,14}$. En contraparte, en los países en desarrollo, además de existir poca evidencia al respecto, parece que los mecanismos penetrante y cerrado representan la misma proporción, o incluso se invierte la tendencia y resulta que el mecanismo penetrante es el preponderante ${ }^{15-17}$. El mecanismo de lesión debe tomarse en consideración incluso para la decisión de proceder o diferir una cirugía, dado que los traumas de energía cinética alta pueden generar lesiones potencialmente mortales que no se evidencian en la revisión primaria ${ }^{18}$.

El diagnóstico de una lesión intraabdominal suele ser difícil por varios factores, entre ellos la ausencia de dolor abdominal pese a una lesión en la cavidad peritoneal o retroperitoneal y la disminución del grado de consciencia por sustancias psicoactivas o a consecuencia del mismo evento traumático ${ }^{19}$. La afirmación anterior es indirectamente apoyada por la alta tasa de laparotomías exploratorias negativas o innecesarias reportadas en la literatura, del $30-40 \%$, pese a la utilización de auxiliares diagnósticos ${ }^{6,20}$.

La decisión de someter a laparotomía a un paciente estable con trauma abdominal ha cambiado radicalmente a lo largo del último siglo y en la actualidad, con el advenimiento de opciones diagnósticas como el lavado peritoneal, la ecografía FAST (Focused Assessment for the Sonographic examination of the Trauma patient), la tomografía computada y la laparoscopia diagnóstica, así como opciones terapéuticas de radiología intervencionista, existe una tendencia creciente a favor del manejo conservador, lo que hace necesaria una laparotomía exploratoria en aproximadamente solo el $20 \%$ de los $\operatorname{casos}^{8,21,22}$. Sin embargo, en nuestro medio, la disponibilidad de estas tecnologías es la excepción, y por ende el tratamiento quirúrgico está basado principalmente en los hallazgos clínicos y en los parámetros hemodinámicos.

Las lesiones encontradas en los pacientes con trauma abdominal son tan diversas como lo son los órganos abdominales y los mecanismos asociados a la lesión. En el trauma cerrado abdominal o contuso, los órganos sólidos como el bazo (40-55\%) y el hígado (35-45\%) se afectan en mayor proporción. Por otro lado, en el trauma penetrante abdominal por arma blanca se describen lesiones principalmente en el hígado (40\%), el intestino delgado (30\%) y el diafragma $(20 \%)$, mientras que el asociado a arma fuego afecta al intestino delgado (50\%), al intestino grueso 
(40\%) y al hígado $(30 \%)^{9}$. Sin embargo, estos datos proceden de países desarrollados, en los que, como se ha documentado previamente, los mecanismos y los agentes de lesión son distintos a los de nuestro contexto, por lo que se vuelve relevante conocer el perfil epidemiológico del trauma abdominal en nuestro medio.

El objetivo del presente estudio es describir y analizar el perfil sociodemográfico, los mecanismos de trauma y las lesiones evidenciadas tras una laparotomía exploratoria, o por medio de tomografía computada en los pacientes sin manejo quirúrgico, en un hospital de referencia en el occidente de México, a fin de servir de base estadística para nuevos análisis que conlleven la implementación de estrategias y políticas de salud pública que eventualmente repercutan en la disminución de los eventos traumáticos y sus secuelas humanas, sociales y económicas.

\section{Método}

En el periodo comprendido del 1 de enero de 1986 al 31 de diciembre de 2017 se analizaron los datos obtenidos del registro GDL-SHOT (Guadalajara Database of Lesions, Statistics On abdominal Trauma) del servicio de cirugía medicina legal, y se incluyeron los pacientes mayores de 15 años con diagnóstico de trauma abdominal. Las variables de estudio fueron la edad, el sexo, el estado civil, la escolaridad, la ocupación, el mecanismo del trauma (penetrante por arma de fuego, arma blanca u objeto punzocortante, y cerrado de cualquier origen), el agente de lesión, el lugar de la lesión, el tratamiento del paciente, las lesiones encontradas, la estancia intrahospitalaria y la mortalidad. En el caso de los pacientes con más de una lesión en el mismo órgano, se consideró la más importante. El estudio fue evaluado y aprobado por el Comité de Ética del Hospital Civil de Guadalajara Fray Antonio Alcalde. El análisis de la información registrada en la base de datos se realizó de manera retrospectiva, por lo que no se solicitó el consentimiento bajo información de los sujetos de estudio.

El diseño del estudio es retrospectivo, observacional y descriptivo de los pacientes ingresados en el servicio de cirugía medicina legal del Hospital Civil de Guadalajara Fray Antonio Alcalde, que es un centro de referencia para trauma en población abierta. Dicho servicio es responsable de los pacientes ingresados con trauma de origen abdominal, y es el único en su tipo en el occidente del país.

\section{Análisis estadístico}

Los datos demográficos, las variables de estudio, los mecanismo del trauma, el lugar de la lesión, el tratamiento y las lesiones encontradas se presentan como proporciones, frecuencias relativas simples y medidas de tendencia central. Todos los datos fueron registrados y analizados en el programa estadístico IBM SPSS Statistics versión 24.

\section{Resultados}

Se evaluaron 5544 pacientes registrados en la base de datos GDL-SHOT a lo largo de 20 años; se excluyeron 255 cuyas lesiones eran secundarias a mecanismos de trauma distintos a los considerados en los criterios de inclusión, como la ingestión de un cuerpo extraño o la introducción de un cuerpo extraño en el recto. Se excluyeron además 328 pacientes por no contar con datos completos de las variables analizadas.

De los 4961 pacientes analizados, el $91.4 \%$ eran de sexo masculino y la edad promedio fue de 28.7 años (RI: $20-34)$. El $52.6 \%$ de los pacientes eran solteros, el $32.2 \%$ estaban casados y el $12.7 \%$ vivían en unión libre. En cuanto al grado de escolaridad, el 38.6\% tenían educación básica, el 39.6\% tenían educación media, el 13\% habían cursado educación media superior y el 3.6\% habían completado la educación superior; el 5.1\% no habían recibido educación de ningún tipo. Respecto a la ocupación, el 31.4\% ejercían algún tipo de oficio, el $18.6 \%$ eran obreros, el $12.5 \%$ eran comerciantes, el $9.7 \%$ eran desempleados, el $8.4 \%$ eran estudiantes, el $6.1 \%$ eran campesinos y el $5 \%$ se dedicaban al hogar.

De los eventos por trauma registrados, 4175 (90\%) ocurrieron en la vía pública y $5 \%$ en el hogar (Fig. 1). En cuanto al mecanismo del trauma, el $67 \%$ correspondió a trauma penetrante y el $33 \%$ a trauma cerrado. El trauma cerrado de abdomen se debió principalmente a accidentes de automotor, con un $73 \%$ de los casos $(n=1189)$, seguido de las agresiones por objeto contundente en el $15 \%(n=249)$; las caídas representaron un muy bajo porcentaje (Fig. 2). Dentro del trauma penetrante de abdomen, las lesiones por arma de fuego fueron las más comunes, con el $41 \%$ de los casos (Fig. 3).

Se realizaron 3630 (73.2\%) laparotomías exploratorias y en $370(10.2 \%)$ de ellas no se evidenciaron hallazgos. En 1849 (50.9\%) se observaron lesiones únicas y en 1411 (38.9\%) hubo lesiones múltiples 


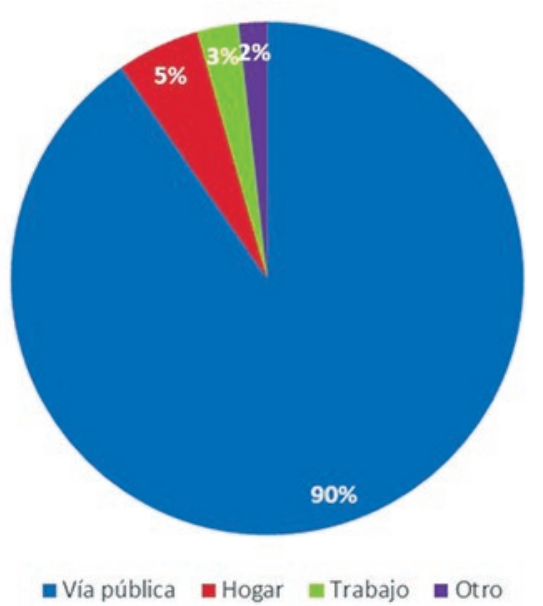

Figura 1. Sitios donde ocurre el trauma abdominal.

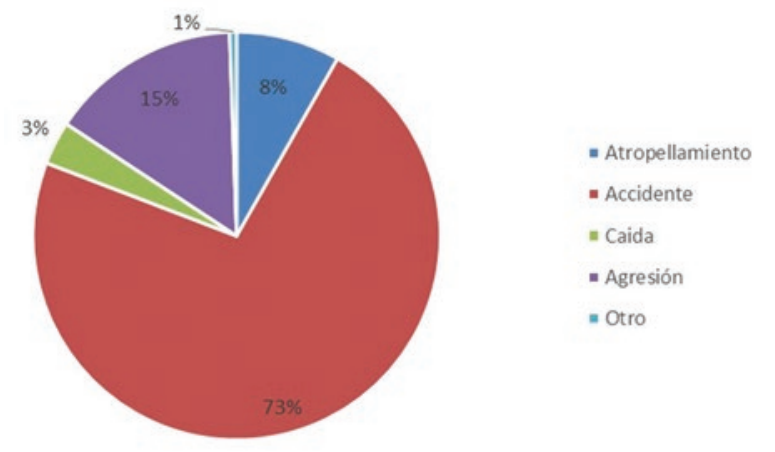

Figura 2. Tipo de lesión en el mecanismo cerrado.

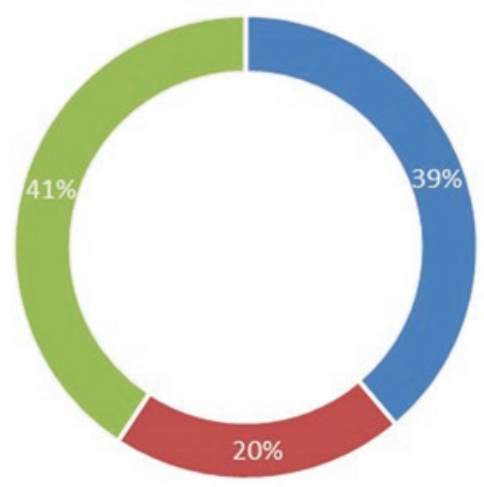

- Arma Blanca - Punzocortante $\|$ Arma de Fuego

Figura 3. Agente de lesión en el mecanismo penetrante.

(Tabla 1). La estancia hospitalaria promedio fue de 6.95 días, con una mortalidad del $6.74 \%$.

En cuanto a las lesiones evidenciadas, los órganos con más frecuencia afectados por trauma son el intestino delgado (20.9\%), el hígado (18.2\%) y el bazo (11.2\%). Al dividir las lesiones por mecanismos de trauma, las lesiones en el bazo (22.9\%), el hígado
(18.7\%) y el intestino delgado (14.2\%) son más frecuentes en el trauma cerrado de abdomen. Respecto al trauma penetrante de abdomen por arma de fuego, resultaron afectados con más frecuencia el intestino delgado (23.6\%), el colon (17.2\%) y el hígado (16.5\%); y por arma blanca, el intestino delgado (23.6\%), el hígado (19.7\%) y el colon (16.4\%) (Tabla 2). En 498 pacientes se encontraron lesiones diafragmáticas, en 428 hubo hematomas retroperitoneales no asociados a una lesión renal y en 284 se hallaron lesiones vasculares abdominales (entre ellas, 13 lesiones aórticas y 46 lesiones de vena cava).

\section{Discusión}

En nuestro estudio, en el análisis de los datos sociodemográficos de los pacientes con trauma abdominal en el occidente de México encontramos que los varones adultos jóvenes son el grupo poblacional mayormente afectado. Aunque los promedios de edad están alrededor de la tercera década de la vida, resulta interesante que, al diferenciar por mecanismo, en el trauma cerrado de abdomen los promedios de edad y el sexo femenino suelen ser mayores que en el trauma penetrante de abdomen. Este dato es semejante a lo reportado en la literatura, y la diferencia es mayor en los países europeos, donde los promedios de edad están alrededor de los 40 años y solo el $80 \%$ de los pacientes son hombres ${ }^{10,20}$. Las lesiones por trauma afectan predominantemente a personas jóvenes, que son el grupo de edad más productivo, lo que de manera invariable repercute en la realidad económica familiar y social, tanto por la falta de productividad como por los costos sanitarios de la atención y las secuelas discapacitantes de los afectados $^{2,23}$. La notoria diferencia que existe entre ambos sexos, con predominio 4:1 del masculino sobre el femenino, se ha explicado por la mayor exposición de los hombres a las armas de fuego y el mayor consumo de sustancias como alcohol y drogas ilegales, lo que los llevaría a tener conductas más agresivas y accidentes por imprudencia ${ }^{16,19}$. Respecto a otras variables sociodemográficas, como el dato de que la mayoría de los pacientes tienen bajo nivel educativo, se ocupan en labores de ingreso escaso o no garantizado, y carecen de relaciones afectivas estables, también se han asociado previamente al consumo de alcohol o sustancias ilícitas y al trauma en general ${ }^{16}$. Sin embargo, consideramos que, además de lo ya mencionado, dado el perfil de nuestro centro, dedicado a la atención a población abierta sin seguridad 
Tabla 1. Variables demográficas y manejo inicial del paciente según el mecanismo del trauma

\begin{tabular}{|c|c|c|c|c|}
\hline & $\begin{array}{l}\text { Trauma } \\
\text { cerrado }\end{array}$ & $\begin{array}{l}\text { Arma de } \\
\text { fuego }\end{array}$ & $\begin{array}{c}\text { Arma blanca u objeto } \\
\text { punzocortante }\end{array}$ & Total, n (\%) \\
\hline Laparotomía exploratoria & $982(30.1 \%)$ & $1012(31.0 \%)$ & $1266(38.8 \%)$ & $3260(65.7 \%)$ \\
\hline $\begin{array}{l}\text { Laparotomía exploratoria sin } \\
\text { hallazgos }\end{array}$ & $54(14.6 \%)$ & $80(21.6 \%)$ & $236(63.8)$ & $370(7.5 \%)$ \\
\hline Observación & $602(45.2 \%)$ & $261(19.6 \%)$ & $468(35.2 \%)$ & $1331(26.8 \%)$ \\
\hline Sexo masculino & $1385(84.5 \%)$ & $1274(94.2 \%)$ & $1876(95.2 \%)$ & $4535(91.4 \%)$ \\
\hline Sexo femenino & $253(15.4 \%)$ & $79(5.8 \%)$ & $94(4.8 \%)$ & $426(8.6 \%)$ \\
\hline Edad & $31.2(20-38)$ & $27.4(20-33)$ & $27.5(20-32)$ & $28.7(20-34)$ \\
\hline Total de pacientes & $1638(33.0 \%)$ & $1353(27.3 \%)$ & 1970 (39.7\%) & 4961 \\
\hline
\end{tabular}

Tabla 2. Lesiones de órganos intraabdominales clasificadas según el mecanismo del trauma

\begin{tabular}{|c|c|c|c|c|}
\hline & $\begin{array}{c}\text { Trauma cerrado } \\
\text { dentro de órgano, } \mathrm{n}(\%) \\
\text { y porcentaje del total de } \\
\text { lesiones }\end{array}$ & $\begin{array}{c}\text { Arma de fuego } \\
\text { dentro de órgano, } n(\%) \\
\text { y porcentaje del total de } \\
\text { lesiones }\end{array}$ & $\begin{array}{c}\text { Arma blanca } \\
\text { dentro de órgano, } \mathrm{n}(\%) \\
\text { y porcentaje del total de } \\
\text { lesiones }\end{array}$ & Total, n (\%) \\
\hline Bazo & 313 (59.3\%) 22.9\% & $93(17.6 \%) 5.2 \%$ & $122(23.1 \%) 7.7 \%$ & $528(11.2 \%)$ \\
\hline Hígado & $256(29.7 \%) 18.7 \%$ & $293(34.0 \%) 16.5 \%$ & $313(36.3 \%)$ 19.7\% & $862(18.2 \%)$ \\
\hline $\begin{array}{l}\text { Vía biliar } \\
\text { extrahepática }\end{array}$ & $14(16.3 \%) 1.0 \%$ & $37(43.0 \%) 2.1 \%$ & $35(40.7 \%) 2.2 \%$ & $86(1.8 \%)$ \\
\hline Páncreas & $74(40.2 \%) 5.4 \%$ & 57 (31.0\%) 3.2\% & $53(28.8 \%) 3.3 \%$ & $184(3.9 \%)$ \\
\hline Estómago & $28(8.6 \%) 2.0 \%$ & $134(41.4 \%) 7.6 \%$ & $162(50.0 \%) 10.2 \%$ & $324(6.9 \%)$ \\
\hline Duodeno & $31(19.1 \%) 2.3 \%$ & $73(45.1 \%) 4.1 \%$ & $58(35.8 \%) 3.6 \%$ & $162(3.4 \%)$ \\
\hline Intestino delgado & 195 (19.7\%) 14.2\% & $418(42.3 \%) 23.6 \%$ & $375(38.0 \%) 23.6 \%$ & $988(20.9 \%)$ \\
\hline Colon & $109(16.2 \%) 7.9 \%$ & 304 (45.1\%) 17.2\% & $261(38.7 \%) 16.4 \%$ & $674(14.2 \%)$ \\
\hline Recto & $63(29.4 \%) 4.6 \%$ & $116(54.2 \%) 6.5 \%$ & $35(16.4 \%) 2.2 \%$ & $214(4.5 \%)$ \\
\hline Glándula adrenal & $7(30.4 \%) 0.5 \%$ & $10(43.5 \%)$ 0.6\% & $6(26.1 \%) 0.4 \%$ & $23(0.5 \%)$ \\
\hline Riñón & 137 (32.8\%) 10.0\% & $139(33.3 \%) 7.8 \%$ & $142(34.0 \%) 8.9 \%$ & $418(8.8 \%)$ \\
\hline Uréter & $8(25.0 \%) 0.6 \%$ & $18(56.3 \%) 1.0 \%$ & $6(18.8 \%) 0.4 \%$ & $32(0.7 \%)$ \\
\hline Vejiga & $131(59.5 \%) 9.6 \%$ & $71(32.3 \%) 4.0 \%$ & $18(8.2 \%) 1.1 \%$ & $220(4.7 \%)$ \\
\hline Útero & $3(50 \%) 0.2 \%$ & $3(50 \%) 0.2 \%$ & $0(0.0 \%) 0.0 \%$ & $6(0.1 \%)$ \\
\hline Trompa & $0(0.0 \%) 0.0 \%$ & $2(66.6 \%) 0.1 \%$ & $1(33.3 \%) 0.1 \%$ & $3(0.1 \%)$ \\
\hline Ovario & $1(20 \%) 0.1 \%$ & $3(60 \%) 0.2 \%$ & $1(20 \%) 0.1 \%$ & $5(0.1 \%)$ \\
\hline Total de lesiones & $1370(29.0 \%)$ & $1771(37.4 \%)$ & $1588(33.6 \%)$ & 4729 \\
\hline
\end{tabular}

social, podría haber un sesgo de selección respecto de pacientes con seguridad social, con trabajos estables, de mayor remuneración económica y probablemente con un mayor nivel educativo.

Uno de los resultados más interesantes de nuestro registro es la baja proporción de traumas cerrados de abdomen que observamos (33\%), lo que contrasta con los datos de reportes europeos $(87-90 \%)^{11,12}$, asiáticos $(64-95 \%)^{23-25}$, australianos $(75-83 \%)^{13}$ y norteamericanos ${ }^{14}$, en los que los accidentes de tráfico y las caídas representan la principal causa de trauma abdominal. En contraste, reportamos una alta proporción de traumas penetrantes de abdomen (67\%), que se corresponde con las realidades de otros países como Brasil $(69-78 \%)^{16,17}$ y Sudáfrica ${ }^{26}(90.2 \%)$. Si bien se reconoce un aumento del trauma penetrante 
globalmente ${ }^{13,20}$, estas diferencias se explican más por las condiciones sociales, económicas y de violencia de países como México y Brasil, además del escaso control sobre el acceso a armas de fuego en la población civil ${ }^{2,5,6,19}$. Como ya se mencionó, la energía del trauma por arma de fuego se relaciona con mayores lesiones y, por ende, con más morbimortalidad, por lo que el control de estas representa una política responsable tanto de seguridad como de salud pública $^{4,24,26}$.

Al comparar nuestros resultados con los de otros países en cuanto a los órganos afectados, encontramos similitudes en el trauma cerrado de abdomen, en el cual el bazo es el órgano más afectado, seguido por el hígado; sin embargo, en nuestro estudio, el intestino delgado se afecta en mayor proporción que el riñón (19.7 vs. $10 \%)$, lo que difiere de otros reportes (10-12 vs. $16-18 \%)^{11,23,27}$. Asimismo, observamos un alto porcentaje de lesiones vesicales $(9.6 \%)$, que se reportan con menor frecuencia en otros estudios (2-4\%) $)^{11,23,27}$. Al evaluar el trauma penetrante por arma de fuego encontramos que los intestinos delgado y grueso son los órganos más afectados, seguidos del hígado, lo que coincide con la mayoría de los reportes previos tanto nacionales como internacionales ${ }^{6,9,15,16}$. Finalmente, el mecanismo por arma blanca u objeto punzocortante representó la mayor cantidad de nuestros pacientes (39.7\%), pero en proporción un menor número de lesiones. En nuestro estudio, los órganos más lesionados fueron el intestino delgado (23.6\%), el hígado $(19.7 \%)$ y el colon (16.4\%), lo que difiere de la experiencia en Norteamérica ${ }^{9}$, donde se lesiona el hígado en primer lugar $(40 \%)$, seguido del intestino delgado $(30 \%)$; de Francia ${ }^{20}$, donde se evidencia una cantidad inusitada de trauma pancreático $(20.4 \%$ de los pacientes); y de Sudáfrica ${ }^{28}$, donde las lesiones colónicas predominan (39\%), junto con las esplénicas (13\%) y las renales (12\%), al incluir pacientes con lesiones en la espalda. Cabe mencionar que, si bien las vísceras huecas son las afectadas con más frecuencia, las lesiones hepáticas se relacionan con mayor mortalidad ${ }^{19}$. Las diferencias aquí expresadas pueden explicarse por los diversos agentes de lesión que participan en el mecanismo cerrado, pues los accidentes de tráfico suelen englobarse sin distinción de las causas ni el mecanismo con que sucedieron. Asimismo, las caídas contribuyen en mayor proporción al trauma cerrado de abdomen en los países desarrollados en comparación con los países en desarrollo, una vez más dejando de lado el análisis del mecanismo con que suceden ${ }^{14}$. Respecto a las heridas penetrantes y perforantes por arma de fuego, es importante considerar el arma utilizada y la balística de esta, que repercuten una vez más en la energía del trauma y, sin embargo, suelen evaluarse dentro del mismo grupo de análisis ${ }^{5}$.

La mortalidad es un dato muy variable en los estudios analizados; en nuestra población encontramos un $6.74 \%$, lo que está dentro de la media, pero puede ser tan alta como el $20-25 \%$ donde predomina el trauma cerrado por accidentes viales ${ }^{11,23,27}$ y tan baja como el $2-6 \%$ donde las lesiones penetrantes por arma blanca son predominantes ${ }^{17,20,28}$. Nuestro dato es ligeramente inferior a lo reportado en México con anterioridad $(7.9-12 \%)^{2,5}$. Una variable que puede ser importante en este sentido, aparte del mecanismo, es la atención o no en centros de trauma especializados, con equipo y personal entrenados, además de protocolos de atención que mejoren el pronóstico de estos pacientes $^{9,23}$.

Una de las limitaciones de nuestro estudio es su diseño retrospectivo, lo que complica la inclusión de variables que surgen a partir de nuevas técnicas, así como la falta de datos completos respecto a las complicaciones y el seguimiento posteriores al alta hospitalaria.

El presente análisis da cuenta de la situación del trauma abdominal en nuestro país y pretende ser un llamado de atención para la implementación de políticas de prevención en tres aspectos principales para la adecuada atención al trauma: el primero, mediante estrategias en formación de recursos humanos y centros hospitalarios especializados en la atención de trauma, además de promover la cultura de prevención de accidentes; el segundo, referente a la infraestructura para el tránsito seguro y la legislación para disminuir la violencia; y el tercero, el control de los vectores como vehículos inseguros y el acceso a armas, en especial las de fuego ${ }^{4}$.

En conclusión, nuestro estudio difiere de lo reportado en otros países, probablemente debido a los distintos mecanismos asociados al trauma, lo que resulta en una disparidad de los órganos afectados. En nuestro medio son menos comunes las lesiones accidentales y son más prevalentes las infligidas. Dada la actual situación de violencia, es importante el desarrollo de investigación relativa al trauma en general y al abdominal en particular, para mejorar el manejo y el pronóstico de los pacientes. Nuestro análisis arroja los resultados del mayor registro de trauma abdominal en México y pretende ser 
cimiento epidemiológico, dada la carencia de este en nuestro país.

\section{Conflicto de intereses}

Los autores declaran que no existen conflicto de intereses que afecten el contenido de este reporte.

\section{Financiamiento}

Los autores declaran que no se contó con financiamiento externo para este estudio.

\section{Responsabilidades éticas}

Protección de personas y animales. Los autores declaran que para esta investigación no se han realizado experimentos en seres humanos ni en animales.

Confidencialidad de los datos. Los autores declaran que en este artículo no aparecen datos de pacientes.

Derecho a la privacidad y consentimiento informado. Los autores declaran que en este artículo no aparecen datos de pacientes.

\section{Bibliografía}

1. DiMaggio C, Ayoung-Chee P, Shinseki M, Wilson C, Marshall G, Lee DC, et al. Traumatic injury in the United States: in-patient epidemiology 20002011. Injury. 2016;47:1393-403.

2. Sánchez-Lozada R, Ortiz-González J, Soto-Villagrán R. Lesiones abdominales por trauma: experiencia de dos años en un hospital de tercer nivel. Cir Gen. 2002;24:201-5.

3. Vos T, Abajobir AA, Abbafati C, Abbas KM, Abate KH, Abd-Allah F, et al Global, regional and national incidence, prevalence, and years lived with disability for 328 diseases and injuries for 195 countries, 1990-2016: a systematic analysis for the Global Burden of Disease Study 2016. Lancet. 2017:390:1211-59.

4. Gosselin RA, Spiegel DA, Coughlin R, Zirkle LG. Injuries: the neglected burden in developing countries. Bull World Health Organ. 2009;87:246.

5. Canseco-Cavazos J, Palacios-Zertuche J, Reyna-Sepúlveda F, Álvarez-Villalobos N, Alatorre-López L, Muñoz-Maldonado G. Epidemiología de las lesiones por proyectil de arma de fuego en el Hospital Universitario "Dr. José Eleuterio González" de la Universidad Autónoma de Nuevo León. Cir Cir. 2017;85:41-8.
6. Pinedo-Onofre JA, Guevara-Torres L, Sánchez-Aguilar JM. Trauma abdominal penetrante. Cir Cir. 2006;74:431-42.

7. Instituto Nacional de Estadística, Geografía e Informática. Registros administrativos de mortalidad; 2016. (Consultado el 16 de enero de 2018.) Disponible en: http://www.inegi.org.mx

8. Leenen LPH. Abdominal trauma: from operative to nonoperative management. Injury. 2009;40:62-8.

9. Committee on Trauma, American College of Surgeons. Advanced trauma life support for doctors-student course manual. $8^{\text {th }}$ ed. Chicago: American College of Surgeons; 2008.

10. Brooks A, Simpson JAD. Blunt and penetrating abdominal trauma. Surgery (Oxford). 2009;27:266-71.

11. Costa G, Tierno SM, Tomassini F, Venturini L, Frezza B, Cancrini G, et al. The epidemiology and clinical evaluation of abdominal trauma. Ann Ital Chir. 2010;81:95-102.

12. Leite $S$, Taveira-Gomes $A$, Sousa $H$. Visceral injury in abdominal trauma: a retrospective study. Act Med Port. 2013;26:725-30.

13. Smith J, Caldwell E, Amours SD, Jalaludin B, Sugrue M. Abdominal trauma: a disease in evolution. ANZ J Surg. 2005;75:790-4.

14. DiMaggio CJ, Avraham JB, Lee DC, Frangos SG, Wall SP. The epidemiology of emergency department trauma discharges in the United States. Acad Emerg Med. 2017;24:1244-56.

15. Senado-Lara I, Castro-Mendoza A, Palacio-Vélez F, Vargas-Ávila AL. Experiencia en el manejo de abdomen agudo de origen traumático en el Hospital Regional "General Ignacio Zaragoza". Cir Cir. 2004;72:93-7.

16. Kruel NF, Oliveira VL, Oliveira VL, Honorato RD, Pinatti BD, Leao FR. Epidemiologic profile of abdominal trauma submitted to laparotomy. Arq Bras Cir Dig. 2007;20:106-10.

17. Lima SO, Cabral FLD, Neto AFP, Mesquita FNB, Feitosa MFG, Santana VRD. Epidemiological evaluation of abdominal trauma victims submitted to surgical treatment. Rev Col Bras Cir. 2012;39:302-6.

18. Prachalias AA, Kontis E. Isolated abdominal trauma: diagnosis and clinical management considerations. Curr Opin Crit Care. 2014;20:218-25.

19. Bordoni PHC, Santos DMMD, Teixeira JS, Bordoni LS. Deaths from abdominal trauma: analysis of 1888 forensic autopsies. Rev Col Bras Cir. 2017;44:582-95.

20. Barbois S, Abba J, Guigard S, Quesada JL, Pirvu A, Waroquet PA, et al. Management of penetrating abdominal and thoraco-abdominal wounds: a retrospective study of 186 patients. J Visc Surg. 2016;153:69-78.

21. Prachalias AA, Kontis E. Isolated abdominal trauma: diagnosis and clinical management considerations. Curr Opin Crit Care. 2014;20:218-25.

22. Como JJ, Bokhari F, Chiu WC, Duane TM, Holevar MR, Tandoh MA, et al. Practice management guidelines for selective nonoperative management of penetrating abdominal trauma. J Trauma. 2010;68:721-33.

23. Arumugam S, Al-Hassani A, El-Menyar A, Abdelrahman H, Parchani A, Peralta $R$, et al. Frequency, causes and pattern of abdominal trauma: a 4 year descriptive analysis. J Emerg Trauma Shock. 2015;8:193-8.

24. Salimi J, Ghodsi M, Nassaji-Zavvarh M, Khaji A. Hospital management of abdominal trauma in Tehran, Iran: a review of 228 patients. Chin $\mathrm{J}$ Traumatol. 2009;12:259-62.

25. Malik R, Atif I, Rashid F, Abbas M. An analysis of 3105 medico legal cases at a tertiary care hospital, Rawalpindi. Pak J Med Sci. 2017;33:926-30.

26. Mnguni MN, Muckart DJJ, Madiba TE. Abdominal trauma in Durban, South Africa: factors influencing outcome. Int Surg. 2012;97:161-8.

27. Helmi I, Hussein A, Ahmed AHA. Abdominal trauma due to road traffic accidents in Qatar. Injury. 2001;32:105-8.

28. Kong VY, Oosthuizen GV, Clarke DL. The spectrum of injuries resulting from posterior abdominal stab wouds: a South African experience. Ann R Coll Surg Engl. 2015;97:269-73. 